\title{
Obstacles risk modeling in aerodromes protection zones
}

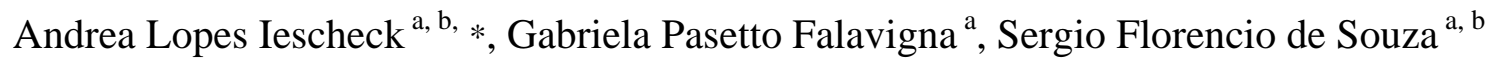 \\ ${ }^{a}$ Remote Sensing Postgraduate Programme, ${ }^{b}$ Department of Geodesy, Federal University of Rio Grande do Sul, Porto Alegre, Rio \\ Grandedo Sul, Brazil, andrea.iescheck@ufrgs.br,gabi_falavigna@hotmail.com,sergio.florencio@ufrgs.br \\ * Corresponding author
}

Keywords: Risk classification, Analytic Hierarchy Process, Spatial analysis

\begin{abstract}
:
This research aims to classify, quantify, and represent the risk of obstacles in the Porto Alegre International Airport protection zone. We evaluated the obstacles related to urban land parcels (plots), buildings, towers, and poles that protrude above the limiting surfaces of the Aerodrome Protection Zone Basic Plan (PBZPA).
\end{abstract}

Obstacles in aerodromes protection zones pose complex problems for air safety and the economy of cities. These violations can reduce or cancel landing procedures, in addition to preventing the expansion of aerodromes and limiting the size of aircraft allowed to operate at airports. The PBZPA consists of limiting surfaces that establish the airspace that must remain clear of obstacles, imposing restrictions on land use within the aerodrome protection zone. Therefore, identifying and quantifying obstacles around airports and knowing each obstacle's risk level is vital for mitigating risks to air operations.

Our research method comprises developing the obstacles risk classification model, generating 3D models, and performing spatial analysis. We used data derived from topographic maps at 1:1,000 scale, orthophotos, lidar, and documents about PBZPA.

The obstacles risk classification model resulted from applying the analytic hierarchy process (AHP) based on a specialist in civil aviation knowledge. We defined four risk factors and prepared a questionnaire to obtain an expert opinion about the relevance of these factors in the analysis and their relative importance in modeling the risk of airport obstacles. We used the same risk factors as Wang et al. (2004), but we adopted a different meaning for the protrusion factor. While Wang et al. (2004) assessed which limiting surface the obstacle protrudes, we analyzed how much each obstacle protrudes above the limiting surfaces. We changed the definition of this factor, considering that obstacles protruding more or less above the same limiting surface will have different associated risk levels. Thus, we defined the risk factors as (a) Distance: distance between the obstacle and the nearest airport runway threshold; (b) Location: in which PBZPA's limiting surface the obstacle is; (c) Type: nature of the obstacle (relief, building, pole, antenna or tower); (d) Protrusion: how much the obstacle protrudes above each limiting surface of the PBZPA.

The resulting model $(R I)$, according to the risk factors and their respective weights, is given by expression (1):

$R I=(0.27 x$ Distance $)+(0.14 x$ Location $)+(0.05 x$ Type $)+(0.54 x$ Protrusion $)$

The values obtained for $R I$ vary according to the risk scale adopted to assess the classes of risk factors. In this study, we adopted a scale of 1 to 5 to analyze the factors' classes, in which number 1 means "very unlikely/very little dangerous" and number 5 means "extremely likely/dangerous." So, values of $R I$ close to 1 represent obstacles that are less dangerous and less likely to compromise air safety. On the other hand, values close to 5 represent more dangerous obstacles, with a high probability of compromising the safety and maintenance of the airport's air operations.

Analyzing the model, we can see that the most significant influence on the risk is how much the obstacle protrudes above the limiting surfaces (54\%), followed by distance (27\%), location (14\%), and type (5\%). The type of obstacle does not significantly influence the risk but improves the model.

To identify and quantify the obstacles, we performed spatial analyses using 3D models of plots, buildings, towers, poles, and PBZPA with an accuracy of $0.5 \mathrm{~m}$. The results showed that from a total of 106,838 plots covering an area of $69.68 \mathrm{~km}^{2}$, 711 plots $(0.66 \%)$ exceeded the limiting restriction surfaces (597 totally and 114 partially), which corresponds to an area of $1.21 \mathrm{~km}^{2}(1.74 \%)$. Also, 264 plots (0.25\%), covering an area of $0.12 \mathrm{~km}^{2}(0.17 \%)$, represent critical areas that should not contain buildings. From a total of 200,573 buildings $\left(27.86 \mathrm{~km}^{2}\right), 7,041$ buildings (3.51\%) exceeded the limiting surfaces (6,707 totally and 334 partially), which correspond to a built-up area of $0.87 \mathrm{~km}^{2}$ (3.12\%). From 344 towers, 11 towers (3.20\%) exceeded the limiting surfaces, and from a total of 51,877 poles, 487 poles (0.94\%) exceeded the limiting surfaces.

Using the obstacles risk classification model, we made a risk map showing the risk associated with the obstacles. We classified the risk as median, high, and very high. In the study area, which covers $174.60 \mathrm{~km}^{2}, 0.58 \mathrm{~km}^{2}(0.33 \%)$ have 
medium-risk obstacles, $1.26 \mathrm{~km}^{2}(0.72 \%)$ have high-risk obstacles, and $0.05 \mathrm{~km}^{2}(0.03 \%)$ have very high-risk obstacles. These results mean that $1.10 \%$ of the study area, considering plots, buildings, poles, and towers, poses risks to airport air operations. Figure 1 shows the risk level of plots, buildings, poles, and towers reached by the PBZPA's limiting surfaces.
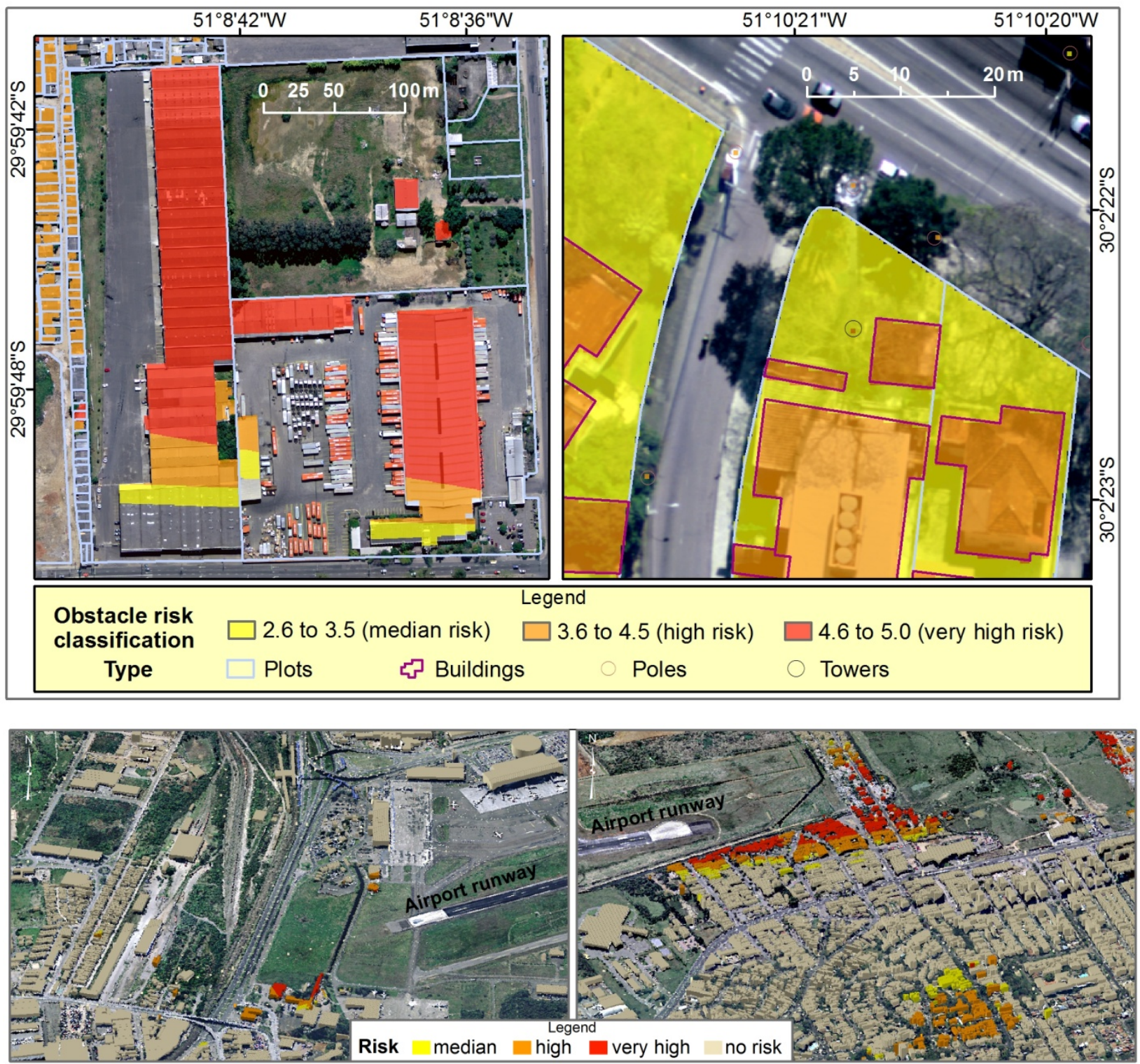

Figure 1. Parts of the obstacle risk classification map and 3D representation of the obstacles risk level.

The methodology proved to be valid for identifying and quantifying obstacles and for obtaining a model to classify the risk of obstacles in aerodrome protection zones. Based on specialist knowledge and using the AHP method, the obstacles risk classification model is valid for any airport and airspace legislation. Considering the risk scores assigned to classes of the risk factors, $R I$ values between 4.6 and 5 mean very or extremely dangerous obstacles, with a high probability of compromising the safety and maintenance of air operations at airports. Learning the risk of obstacles to airspace safety is essential to ensure an acceptable operational safety level at airports. Thus, the airport managers should use the risk modeling of obstacles in aerodrome protection zones to assess risk situations, assisting in the effective management of obstacles and approaches to risk mitigation actions for airspace operations. 